\title{
Technique for estimating perceptual noise in categorization tasks
}

\author{
LEOLA A. ALFONSO-REESE \\ University of California, Santa Barbara, California \\ and San Diego State University, San Diego, California
}

\begin{abstract}
A procedure, based on the method of adjustment, for estimating perceptual noise is presented. The resulting estimates can be used to obtain a better understanding of categorization behavior, to answer questions about the distributional form of perceptual noise, and to test for perceptual independence. All three applications are demonstrated in a categorization task involving two categories.
\end{abstract}

When an observer repeatedly perceives an object, that observer's perception of the object varies from trial to trial. This is partly due to external noise factors such as varying amounts of light in the room in which the object is being perceived, and partly due to noise in the observer's perceptual system. The latter, called perceptual noise, is often ignored by categorization researchers (although Ashby and colleagues traditionally model perceptual noise in categorization and identification tasks [e.g., Alfonso-Reese, Ashby, \& Brainard, in press; Ashby \& Lee, 1992; Ashby $\&$ Maddox, 1990]). Sometimes noise should be ignoredif, for example, the stimuli are highly discriminable and if experimental conclusions are not based on tiny effects. However, in areas such as perceptual categorization, experimenters often use stimuli that are confusable, in order to obtain data that help distinguish between alternative categorization models. In these situations, perceptual noise is likely to play a significant role.

In order to evaluate effects of perceptual noise, we need some way to estimate it. In this article, I describe a technique that yields rough estimates of perceptual noise at various locations in the stimulus space. This technique is a variation of MacAdam's (1942) procedure for estimating sensitivity to color differences, more commonly known as the method of adjustment (Fechner, 1860; Gescheider, 1985). ${ }^{1}$ I apply this technique to a categorization experiment by Ashby, Alfonso-Reese, Turken, and Waldron (1998). This particular application shows how perceptual noise could systematically bias a human classifier's response pattern.

This article is based on portions of the PhD dissertation submitted to the University of California, Santa Barbara, in 1996 and on an unpublished Technical Report (Alfonso-Reese, 1997) available from Indiana University at Bloomington. I thank my teachers, F. Gregory Ashby, David H. Brainard, and John M. Foley, for their advice on this work and on other experiments reported in my dissertation. Correspondence concerning this article should be addressed to L. A. Alfonso-Reese, Department of Psychology, 5500 Campanile Drive, San Diego State University, San Diego, CA 92182-4611 (e-mail: leola@alum.mit.edu).
Besides serving as a tool for obtaining a better understanding of categorization data, this technique can be used to investigate other aspects of perceptual noise. One application is to examine the distributional form of noise, and another is to determine whether components of a stimulus are perceptually independent-that is, whether components of a stimulus do not systematically affect one another. Until now, researchers have had to rely on assumptions about the distributional form of perceptual noise (e.g., Ashby \& Maddox, 1990), and they have had to devise clever modeling and mathematical approaches for investigating issues such as perceptual independence (e.g., Ashby \& Lee, 1991; Kadlec \& Townsend, 1992). The present technique provides a direct method for studying these complex issues.

\section{How to Estimate Perceptual Noise}

Suppose an experimenter wants to estimate perceptual noise induced by the viewing of line stimuli that vary along two dimensions, such as length and orientation. The procedure is as follows:

1. Select a point in the two-dimensional stimulus space at which an estimate of perceptual noise is to be obtained. This is called a reference point. Also, generate a stimulus corresponding to this reference point.

2. Generate a random sample of stimuli from a circular uniform distribution around the reference point, letting the spread of the sample be fairly large. These are called variable stimuli.

3. Present the reference stimulus on one side of a computer display and a variable stimulus on the other side of the display. Have the observer adjust the variable stimulus until it matches the referent. Repeat this process until all randomly generated variable stimuli have been adjusted. To minimize external noise factors, the stimuli should be presented in a dimly lit room with high contrast, and the mechanism for adjusting stimuli should be carefully constructed. (See the experimental procedure for an example of a carefully constructed adjustment mechanism.) 
The resulting distribution of adjusted points represents perceptual noise. From this distribution, a covariance matrix can be computed. ${ }^{2}$ The covariance matrix $(\Sigma)$ contains an estimate of perceptual noise along each dimension $\left(\sigma_{\text {len }}, \sigma_{\text {orien }}\right)$ and correlation between the two dimensions $(\rho)$ at the selected reference point in the stimulus space:

$$
\sum=\left[\begin{array}{ll}
\sigma_{\text {len }}^{2} & \rho \sigma_{\text {len }} \sigma_{\text {orien }} \\
\rho \sigma_{\text {len }} \sigma_{\text {orien }} & \sigma_{\text {orien }}^{2}
\end{array}\right]
$$

In practice, an experimenter will probably want to estimate noise at multiple locations in relevant regions of the stimulus space.

It is important to acknowledge that the technique described here gives only a rough estimate of perceptual noise, since other noise factors have been minimized but not eliminated. For example, noise resulting from memory loss is reduced by presenting the reference stimulus and the variable stimulus simultaneously. However, memory is still required, since the observer must turn his/her attention from one side of the display to the other in order to make a comparison judgment. Thus, while use of the phrase perceptual noise is somewhat of a misnomer, this term captures the essence of what is actually estimated. It is also important to note that this technique should be used only with observers who are experienced at viewing the stimuli of interest. Otherwise, the range of final noise adjustments may change with practice because of perceptual learning (Goldstone, 1994).
Next, I describe a categorization study followed by a corresponding perceptual noise experiment.

\section{Previous Categorization Study}

In a previous study, my colleagues and I (Ashby et al., 1998) conducted an experiment consisting of two conditions, only one of which is discussed here. The observer's task was to learn to correctly classify 500 line stimuli, varying in length and orientation, into one of two categories, $A$ or $B$. The length and orientation of each stimulus was determined by randomly drawing a point from one of two bivariate normal distributions. Half of the stimuli were picked from the category $A$ distribution and half from the category $B$ distribution. ${ }^{3}$ The entire sample of stimuli, represented as points in space, is depicted in Figure 1. The category $A$ stimuli are plotted as plus symbols, and the category $B$ stimuli are plotted as circles.

According to decision-bound theory (Ashby, 1992), on which this experiment is based, an observer in this task will learn to separate the stimulus space into category regions. A boundary separating the regions represents a categorization rule. The optimal rule in Figure 1 is represented by the solid line. An ideal observer ${ }^{4}$ using the optimal rule will respond " $A$ " to stimuli above the line and " $B$ " to stimuli below the line. Doing this, the observer can respond correctly to $75.9 \%$ of the stimuli. Using a categorization rule based on just length or orientation of the line, an ideal observer can respond correctly to just $64.1 \%$ or $72.7 \%$ of the stimuli, respectively. These dimensional rules are depicted in Figure 1 as horizontal or vertical dotted lines.

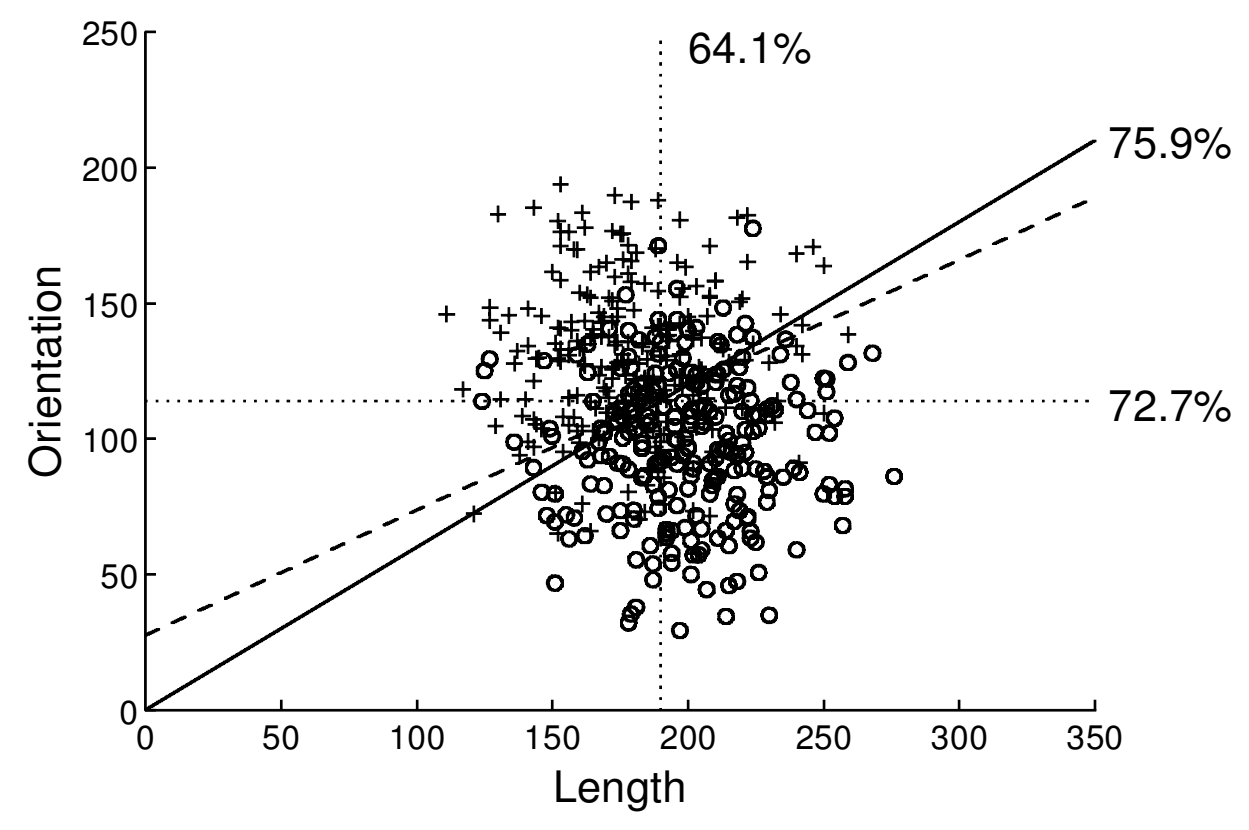

Figure 1. Experimental design in the Ashby et al. (1998) study showing stimuli and decision bounds. The category $A$ stimuli are plotted as plus symbols, and the category $B$ stimuli are plotted as circles. The optimal rule yields $75.9 \%$ correct, and the best dimensional rule, the horizontal line, yields $72.7 \%$ correct. The dashed line represents a typical participant's categorization rule. 
In this categorization study, we found that observers' decision boundaries were systematically biased away from the optimal rule and toward a rule on orientation - that is, toward a horizontal dimensional rule. For example, a typical participant's rule is represented by the dashed line in Figure 1. Thus, observers did not use an optimal strategy, nor did they base their categorical decision on just one dimension of the stimulus set. How can perceptual noise help one to interpret these results? The following experiment addresses this question.

\section{EXPERIMENT}

\section{Participants}

Three female and two male graduate students at the University of California at Santa Barbara participated in this experiment. Each participant received $\$ 8$ for the first hour plus $\$ 8$ per 45 min beyond the first hour. All the participants were experienced at categorizing computergenerated line stimuli because they had previously participated in a categorization experiment using the stimulus set described in Figure 1. Details of this prior categorization experiment can be found in Alfonso-Reese (1996).

\section{Method}

Procedure. The participant's task was to adjust the stimulus on the right side of the screen (the variable stimulus) until it exactly matched the stimulus on the left side of the screen (the referent). The procedure continued until 300 stimuli had been adjusted.

Stimuli and Materials. Stimuli were generated according to guidelines specified by the noise estimation technique described above. Six reference points were chosen. These reference points and variable stimuli corresponding to one reference point are depicted in Figure 2. Each point $(x, y)$ was converted to a line stimulus on a computer monitor, where $x$ determined length of the line and $y$ determined orientation. In particular, $x$ and $y$ are scaled by .85 and $\pi / 550$ respectively, to generate a line $.85 x$ pixels long and rotated $\pi / 550 y$ radians counterclockwise from the horizontal position. 5 The stimuli were presented on a SuperMac Technology 17T Color Display driven by a Power Macintosh 7100 running psychophysics software (Brainard, 1997) within MATLAB (1995). Each participant sat 45 in. away from the monitor. Each referent was rotated either $26.2^{\circ}$ or $49.1^{\circ}$ counterclockwise from horizontal, and the visual angle based on the length of these lines was $2.2^{\circ}, 2.9^{\circ}$, or $3.7^{\circ}$. Resolution of the monitor was $768 \times 1,024$. The image area subtended $14.85^{\circ}$ horizontally by $11.62^{\circ}$ vertically. To minimize external noise, the stimuli were presented with high contrast (white lines on a black background) in a dimly lit room. To eliminate jaggedness of the line stimuli due to limited resolution of the monitor, smooth lines were created with an anti-alias line generation program.

The mechanism used for adjusting the stimuli was as follows: Four arrows on an extended keyboard were set apart from the rest of the keys. Three keys with an arrow pointing left, down, and right were positioned in a horizontal row, and one key pointing up was positioned above the down arrow key. When the up and down arrows were pressed, the orientation of the line increased and decreased, respectively. When the left and right arrows were pressed, the length of the line decreased and increased, respectively. If the participant tapped a key, the line changed by one unit amount, \pm 1 pixel for length or $\pm 0.327^{\circ}$ for orientation. Tiny adjustment amounts were chosen so that one unit of change would be imperceptible to the observer. If the participant held a key down for a short time, the line changed slowly. If the participantheld the key down for a long time, the line changed quickly. When a key was released, adjustment size returned to just one unit per keypress. Finally, the participant indicated that he/she was finished making adjustments by pressing the space bar. To minimize accidental space bar hits, one arrow key had to be pressed before the computer accepted a space bar press.

\section{Results and Discussion}

Perceptual noise estimates and interpretation of categorization data. A scatterplot of final locations of all the adjusted stimuli for Participant 1 is depicted in Figure 3. Two symbols are used in this scatterplot, solely to help the reader separate members of adjacent clusters. Each cluster represents a distribution estimate of perceptual noise. The large elliptical shapes surrounding each cluster are approximate $99 \%$ distribution contours. A circle represents equal noise in the length and orientation dimensions. A horizontal ellipse represents greater noise in perceived length, and a vertical ellipse represents greater noise in perceived orientation. Note that some of the contours in Figure 3 are slightly oblique, rather than perpendicular to an axis. The amount of obliqueness corresponds to the amount of correlation between noise along the two dimensions. Later in this paper, I test whether these correlations are significant.

Distribution contours for the remaining 4 participants are similar to those depicted in Figure 3. Standard deviations for length, $\sigma_{\mathrm{L}}$, and orientation, $\sigma_{\mathrm{O}}$, and correlation estimates, $\rho$, obtained for each observer are listed in Table 1. A repeated-measures test comparing the mean standard deviations of perceptual noise for each participant along the length and orientation dimensions indicates that average perceptual noise for length is greater than average perceptual noise for orientation $[t(4)=5.75, p<.01]$. In fact, the estimated perceptual noise for length exceeds that of orientation for 28 of the 30 distributions (see Table 1).

It appears that each observer's perceptual system is more sensitive to change along the orientation dimension for stimuli in this region of the stimulus space. This is not surprising, since we know that Weber's fraction for line stimuli is .029; that is, perceptual noise along the length dimension increases as line length increases. Thus, noise along the length dimension might exceed noise along the orientation dimension for long lines. For short lines, noise along length might be less than noise along orientation. Figure 3 shows a hint of this reversal with the short 

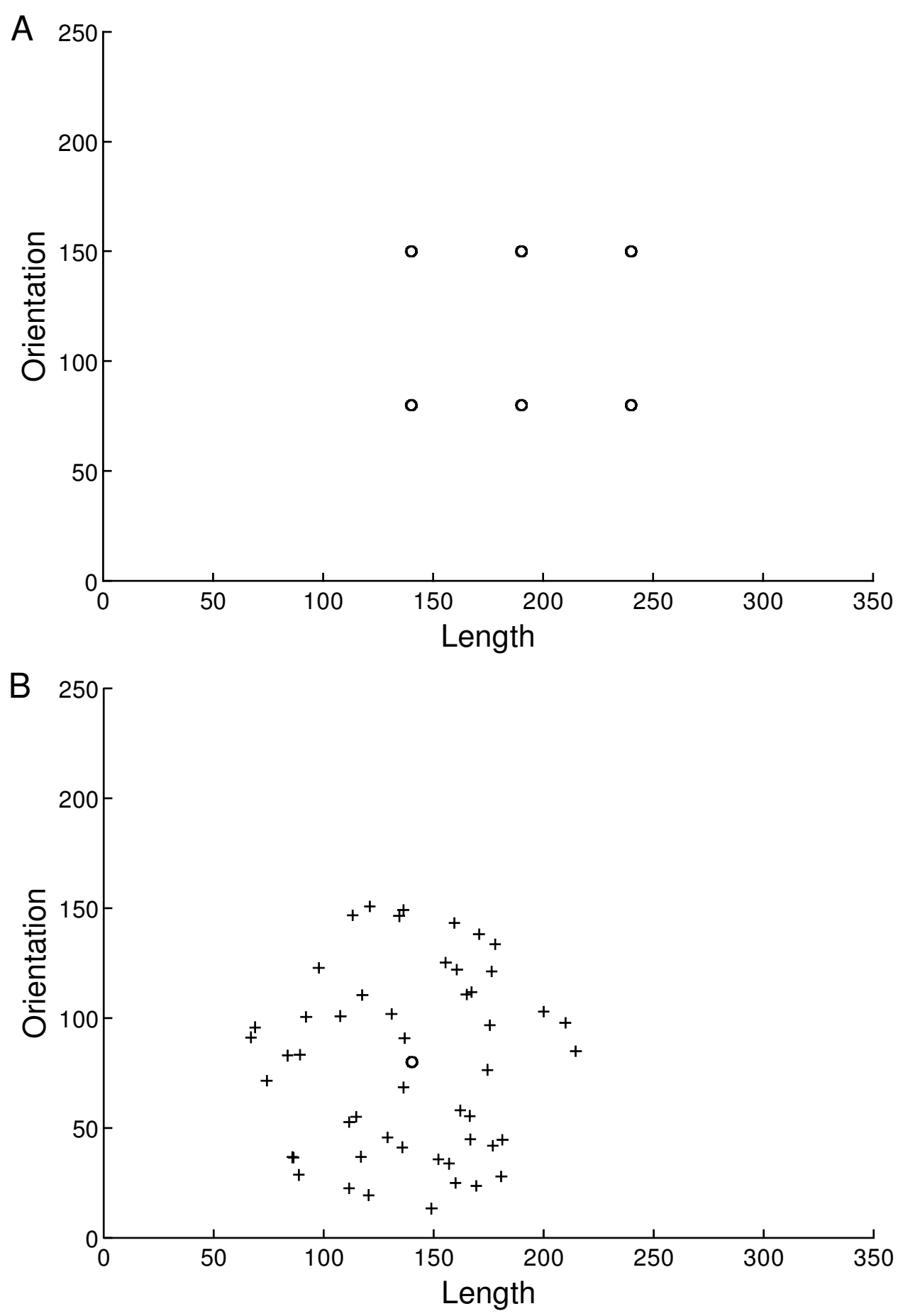

Figure 2. Two-dimensional representation of stimuli used in the experiment. (A) Six reference points. (B) Variable stimuli (represented by plus symbols) for one of the reference points (represented by a circle).

stimuli-those that are 140 units long. Furthermore, we know that human sensitivity to line length depends on orientation and vice versa (Orban, Vandenbussche, \& Vogels, 1984). Altogether, the resulting noise estimates agree with expectations based on previous research.
How do these noise estimates help us understand categorization behavior? Given the noise pattern in Figure 3 , we might expect a human classifier to rely more on orientation than on length information because the perceptual system is more sensitive to changes along orien- 
tation. More attention on orientation might result in a bias toward horizontal categorization rules, pushing the learned categorization boundary away from the optimal oblique angle and toward a suboptimal horizontal rule. Ultimately, a classifier might use a boundary that is shallower than optimal, like the dashed line in Figure 1.

This hypothesis is obviously speculative. An entirely different explanation for the relationship between perceptual noise and categorization behavior is as follows: Because the observer learned to use a nearly optimal categorization rule, the observer learned to discriminate lines of similar orientation better than lines of similar length. Thus, the noise estimates merely reflect perceptual learning. This argument suggests an interesting study comparing pre- and postcategorization meaures of perceptual noise. Regardless of the correct theoretical explanation for the relationship between my perceptual noise estimates and categorization behavior, the present results demonstrate how the noise estimation technique can assist one's understanding of categorization behavior. ${ }^{6}$

\section{OTHER APPLICATIONS OF THE NOISE ESTIMATION TECHNIQUE}

In addition to serving as a tool for studying categorization, the noise estimation technique can be used to investigate questions about the representation of perceptual noise. The following tests check for normality of the dis- tribution of percepts and for perceptual independence of line length and orientation.

\section{Tests of Normality}

Mardia's $(1970,1974)$ skewness and kurtosis tests of multivariate normality were performed on all six distributions for each participant. The hypothesis that the perceptual noise estimate is bivariate normal was rejected for 9 out of 30 distributions using $\alpha=.05 .{ }^{7}$ (See Table 2.) For each participant, the normality assumption was upheld for at least two thirds of the noise distributions. Therefore, researchers seem justified in assuming that perceptual noise is normally distributed for line stimuli varying in length and orientation.

\section{Tests of Perceptual Independence}

According to Ashby and Townsend (1986), two components of a stimulus, such as length and orientation, are perceptually independent if and only if the perceptual effects of each component are statistically independent—-that is, if the bivariate distribution is a product of the marginals. In the special case where the distribution of percepts is normally distributed, perceptual independence holds if the two components are uncorrelated. On the basis of these concepts, I tested for perceptual independence by first determining whether noise along the length and orientation dimensions was uncorrelated. I performed likelihood ratio tests (Wilks's $\Lambda$ ) on all six distributions for each partici-

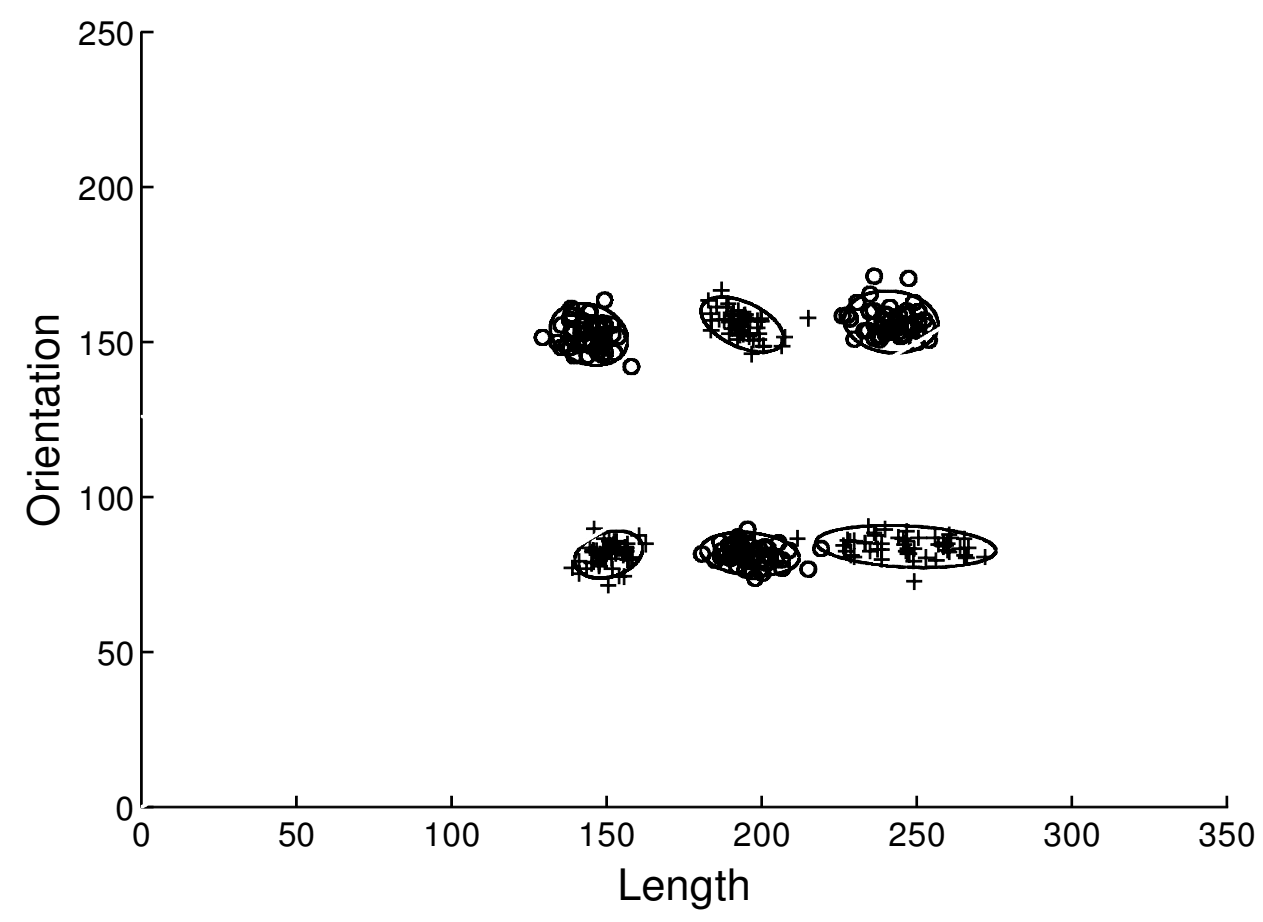

Figure 3. Noise distributions represented by clusters of points for Participant 1 in the experiment. Two symbols are used solely to help the reader separate members of adjacent clusters. The elliptical shapes around each cluster are approximate $99 \%$ distribution contours. 
Table 1

Standard Deviation and Correlation Estimates of Perceptual Noise

\begin{tabular}{|c|c|c|c|c|c|c|c|}
\hline \multirow{2}{*}{$\begin{array}{c}\text { Reference } \\
\text { Point }\end{array}$} & \multirow{2}{*}{$\begin{array}{c}\text { Noise } \\
\text { Parameter }\end{array}$} & \multicolumn{5}{|c|}{ Participant } & \multirow[b]{2}{*}{ Means } \\
\hline & & 1 & 2 & 3 & 4 & 5 & \\
\hline \multirow[t]{3}{*}{$(140,80)$} & $\sigma_{\mathrm{L}}$ & 3.61 & 4.28 & 6.27 & 3.15 & 3.74 & 4.21 \\
\hline & $\sigma_{\mathrm{O}}$ & 2.51 & 4.91 & 5.52 & 2.59 & 2.76 & 3.66 \\
\hline & $\rho$ & .26 & -.18 & -.30 & -.17 & -.44 & -.17 \\
\hline \multirow[t]{3}{*}{$(190,80)$} & $\sigma_{\mathrm{L}}$ & 5.24 & 6.21 & 8.25 & 5.75 & 4.30 & 5.95 \\
\hline & $\sigma_{\mathrm{O}}^{\mathrm{L}}$ & 2.29 & 4.37 & 6.61 & 2.53 & 2.74 & 3.71 \\
\hline & $\rho$ & -.17 & -.10 & -.50 & -.31 & -.30 & -.28 \\
\hline \multirow[t]{3}{*}{$(240,80)$} & $\sigma_{\mathrm{L}}$ & 9.54 & 6.65 & 9.04 & 5.48 & 6.24 & 7.39 \\
\hline & $\sigma_{\mathrm{O}}$ & 2.25 & 5.51 & 4.14 & 2.54 & 2.92 & 3.47 \\
\hline & $\rho$ & -.20 & .36 & -.38 & -.06 & -.50 & -.16 \\
\hline \multirow[t]{3}{*}{$(140,150)$} & $\sigma_{\mathrm{L}}$ & 4.11 & 4.00 & 6.63 & 3.88 & 3.72 & 4.47 \\
\hline & $\sigma_{\mathrm{O}}$ & 3.29 & 4.28 & 5.12 & 2.72 & 3.33 & 3.75 \\
\hline & $\rho$ & -.19 & .17 & .17 & -.09 & -.26 & -.04 \\
\hline \multirow[t]{3}{*}{$(190,150)$} & $\sigma_{\mathrm{L}}$ & 4.39 & 5.93 & 7.00 & 4.51 & 4.08 & 5.18 \\
\hline & $\sigma_{\mathrm{O}}$ & 2.91 & 3.95 & 4.71 & 3.01 & 3.83 & 3.68 \\
\hline & $\rho$ & -.48 & -.14 & -.19 & .16 & -.57 & -.24 \\
\hline \multirow[t]{3}{*}{$(240,150)$} & $\sigma_{\mathrm{L}}$ & 4.99 & 5.44 & 8.90 & 5.40 & 4.64 & 5.87 \\
\hline & $\sigma_{\mathrm{O}}$ & 3.29 & 3.78 & 3.88 & 2.30 & 2.90 & 3.23 \\
\hline & $\rho$ & -.08 & -.13 & .02 & -.08 & -.43 & -.14 \\
\hline \multirow[t]{3}{*}{ Means } & $\bar{\sigma}_{\mathrm{L}}$ & 5.31 & 5.42 & 7.68 & 4.69 & 4.45 & 5.51 \\
\hline & $\bar{\sigma}_{\mathrm{O}}$ & 2.76 & 4.47 & 5.00 & 2.62 & 3.08 & 3.58 \\
\hline & $\bar{\rho}$ & -.14 & .00 & -.20 & -.09 & -.42 & -.17 \\
\hline
\end{tabular}

pant. The hypothesis that the covariance of length and orientation is zero was rejected for 10 out of 30 distributions using $\alpha=.05$. (See Table 2.) Out of the 21 distributions that were found to be normal in the previous set of tests, 14 of them satisfy perceptual independence. From Table 2, we see that perceptual independence does not consistently hold for any individual participant or any single stimulus. This result is similar to previous findings of Ashby and Lee (1991). Although Ashby and Lee (1991) were able to test for perceptual independence, their methods were more complex than those used in the present study. For exam- ple, to estimate the perceptual noise of nine stimuli, they performed extensive model fits, where the most general model used 53 parameters to fit 91 data points. When so many parameters are required, findings may not be as accurate as those obtained with the noise estimation technique.

\section{SUMMARY AND CONCLUSIONS}

In this study I have borrowed a classical technique from the color perception literature and introduced it to the cat-

Table 2

Skewness $(S)$ and Kurtosis $(K)$ Values for Tests of Normality and Wilks's Lambda $(\Lambda)$ Values for Tests of Zero Correlation

\begin{tabular}{ccccccc}
\hline \multirow{2}{*}{$\begin{array}{c}\text { Reference } \\
\text { Point }\end{array}$} & Statistic & 1 & 2 & 3 & \multicolumn{5}{c}{ Participant } \\
\cline { 2 - 6 }$(140,80)$ & $S$ & 0.268 & 0.830 & 0.214 & 0.925 & 0.461 \\
& $K$ & 8.453 & 9.209 & 7.701 & 8.801 & 7.345 \\
& $\Lambda$ & $0.931^{\mathrm{I}}$ & 0.966 & $0.908^{*}$ & $0.972^{\mathrm{I}}$ & $0.809^{*}$ \\
$(190,80)$ & $S$ & 0.692 & $1.170^{*}$ & $6.785^{*}$ & $1.093^{*}$ & $1.444^{*}$ \\
& $K$ & 8.386 & 7.615 & $18.08^{*}$ & 9.056 & 8.514 \\
& $\Lambda$ & $0.970^{\mathrm{I}}$ & 0.990 & $0.748^{*}$ & $0.903^{*}$ & $0.910^{*}$ \\
$(240,80)$ & $S$ & 0.623 & $1.833^{*}$ & 0.223 & 0.578 & 0.843 \\
& $K$ & 8.321 & $13.88^{*}$ & 6.869 & 8.094 & 9.397 \\
$(140,150)$ & $\Lambda$ & $0.961^{\mathrm{I}}$ & 0.874 & $0.854^{*}$ & $0.996^{\mathrm{I}}$ & $0.747^{*}$ \\
& $S$ & 0.418 & 0.049 & 0.063 & 0.327 & 0.264 \\
& $K$ & 7.337 & 7.167 & $6.032^{*}$ & 8.173 & 9.070 \\
$(190,150)$ & $\Lambda$ & $0.965^{\mathrm{I}}$ & $0.971^{\mathrm{I}}$ & 0.971 & $0.993^{\mathrm{I}}$ & $0.934^{\mathrm{I}}$ \\
& $S$ & $1.874^{*}$ & 0.505 & 0.333 & $1.126^{*}$ & 0.374 \\
& $K$ & $12.01^{*}$ & 7.685 & 8.157 & 9.491 & 6.582 \\
$(240,150)$ & $\Lambda$ & $0.765 *$ & $0.979 \mathrm{I}$ & $0.964^{\mathrm{I}}$ & 0.973 & $0.677^{*}$ \\
& $S$ & $1.516^{*}$ & 0.263 & 0.059 & 0.675 & 0.464 \\
& $K$ & 8.829 & 7.053 & 7.859 & 7.902 & 8.656 \\
& $\Lambda$ & 0.993 & $0.984^{\mathrm{I}}$ & $1.000^{\mathrm{I}}$ & $0.994^{\mathrm{I}}$ & $0.814^{*}$ \\
\hline * Significant at & $\alpha=05$ & ISatisfied perceptual independence. &
\end{tabular}

*Significant at $\alpha=.05 \quad$ ISatisfied perceptual independence. 
egorization community as a tool for estimating perceptual noise. I have shown how this technique can be used to aid the interpretation of data, using an example from Ashby et al. (1998). I argue that response biases found in the categorization data could be partly due to systematic patterns of perceptual noise. Alternatively, perceptual noise could systematically vary as a consequence of category learning. Either way, researchers could benefit from the proposed technique to closely examine the relationship between perceptual noise and category learning.

Another application for the noise estimation technique is to investigate the distributional form of perceptual noise. Until now, researchers have typically assumed a multivariate normal distribution and then estimated the noise levels via model-fitting procedures. The new approach suggested in this paper involves statistical tests of representation assumptions based on direct, experimentally derived estimates of perceptual noise. A specific conclusion of the present study is that perceptual noise for line stimuli varying in length and orientation may be reasonably represented by a bivariate normal distribution. Also, perceptual independence is inconsistently satisfied in the tested region of the stimulus space.

\section{REFERENCES}

Alfonso-Reese, L. A. (1996). Dynamics of category learning. Unpublished doctoral dissertation, University of California, Santa Barbara. Alfonso-Reese, L. A. (1997). On the dangers of ignoring noise in highlevel perception experiments (Research Report No. 196). Bloomington: Indiana University, Cognitive Science Program.

Alfonso-Reese, L. A., Ashby, F. G., \& Brainard, D. H. (in press). What makes a categorization task difficult? Perception \& Psychophysics.

AshBY, F. G. (1992). Multidimensionalmodels of categorization. Hillsdale, NJ: Erlbaum.

Ashby, F. G., Alfonso-Reese, L. A., Turken, A. U., \& Waldron, E. M. (1998). A neuropsychological theory of multiple systems in category learning. Psychological Review, 105, 442-481.

Ashby, F. G., \& Lee, W. W. (1991). Predicting similarity and categorization from identification. Journal of Experimental Psychology: General, 120, $150-172$.

Ashby, F. G., \& Lee, W. W. (1992). On the relationship between identification, similarity, and categorization: Reply to Nosofsky and Smith (1992). Journal of Experimental Psychology: General, 121, 385-393.

AshBY,F. G., \& MADDOX, W. T. (1990). Integrating information from separable psychological dimensions. Journal of Experimental Psychology: Human Perception \& Performance, 16, 598-612.

AshBy, F. G., \& Townsend, J. T. (1986). Varieties of perceptual independence. Psychological Review, 93, 154-179.

Brainard, D. H. (1997). The Psychophysics Toolbox. Spatial Vision, 10, 433-436.

Fechner, G. T. (1860). Elemente der Psychophysik. Leipzig: Breitkoph \& Härtel.

GeSCheIDER, G. A. (1985). Psychophysics: Method, theory, and application. Hillsdale, NJ: Erlbaum.

GoLDSTONE, R. (1994). Influences of categorization on perceptual discrimination. Journal of Experimental Psychology: General, 123, 178200.
KADLEC, H. \& TownSEND, J. T. (1992). Implications of marginal and conditional detection parameters for the separabilities and independence of perceptual dimensions. Journal of Mathematical Psychology, 36, 325374.

MacAdam, D. L. (1942). Visual sensitivities to color differences in daylight. Optical Society of America, 32, 247-274.

MARDIA, K. V. (1970). Measures of multivariate skewness and kurtosis with applications. Biometrika, 57, 519-530.

Mardia, K. V. (1974). Applications of some measures of multivariate skewness and kurtosis for testing normality and robustness studies. Sankhya: Series B, 36, 115-128.

Matlab (Macintosh Version 4.2c.1) [Computer software]. (1995). Natick, MA: The MathWorks.

Orban, G. A., Vandenbussche, E., \& Vogels, R. (1984). Human orientation discrimination tested with long stimuli. Vision Research, 24, 121-128.

\section{NOTES}

1. I thank David H. Brainard for suggesting this procedure.

2 . Note that because two stimuli are presented simultaneously in each trial, the resulting distribution of adjusted stimuli reflects noise contributed by both the referent and the adjusted stimulus. Thus, to obtain an estimate corresponding to the adjusted stimulus alone, the covariance matrix must be scaled by one half. This manipulation assumes that noise for each stimulus pair is independent.

3 . The category $A$ distribution is specified by the mean $\mu_{\mathrm{A}}$ and covariance matrix $\Sigma$ while the category $B$ distribution is specified by the mean $\mu_{\mathrm{B}}$ and the same covariance matrix $\Sigma$ :

$$
\begin{gathered}
\mu_{A}=\left[\begin{array}{l}
180 \\
130
\end{array}\right], \\
\mu_{B}=\left[\begin{array}{c}
200 \\
98
\end{array}\right],
\end{gathered}
$$

and

$$
\Sigma=\left[\begin{array}{cc}
729 & 0 \\
0 & 729
\end{array}\right]
$$

4. An ideal observer is one who is not subject to perceptual or criterial noise. For this observer, the perceptual space is exactly the same as the stimulus space.

5 . The scale factors, .85 and $\pi / 550$, were chosen so that the computergenerated stimuli had the same visual angle and orientation as those used in the Ashby et al. (1998) study.

6 . One concern regarding this technique is whether participants systematically undershoot or overshoot the point of subjective equality, depending upon the starting point. A preliminary analysis revealed no significant difference in the final orientation values for the two groups (high vs. low starting value) $(p>.6)$, and no significant difference in the final length values for the high versus low groups $(p>.09)$. Although the method of adjustment is prone to influences from the observer's bias, such as adjusting length in accordance with stimulus presentation, this potential effect did not largely affect the present study.

7. The use of $\alpha=0.5$ in the normality and perceptual independence tests reflects a compromise between two goals: (1) to avoid making a Type I error, given the large number of tests being performed (i.e., choosing a small alpha), and (2) to perform a strong test of normality and independence (i.e., using a large alpha).

(Manuscript received July 10, 2000; revision accepted for publication February 7, 2001.) 